\title{
Effects of Neurotrophins on Synaptic Protein Expression in the Visual Cortex of Dark-Reared Rats
}

\author{
Tiziana Cotrufo, Alessandro Viegi, Nicoletta Berardi, Yuri Bozzi, Laura Mascia, and Lamberto Maffei \\ Scuola Normale Superiore, 56126 Pisa, Italy, Istituto di Neuroscienze, Consiglio Nazionale delle Ricerche, 56100 Pisa, Italy, and Dipartimento di Psicologia, \\ Universita' di Firenze, 50123 Firenze, Italy
}

Total lack of visual experience [dark rearing (DR)] is known to prolong the critical period and delay development of sensory functions in mammalian visual cortex. Recent results show that neurotrophins (NTs) counteract the effects of DR on functional properties of visual cortical cells and exert a strong control on critical period duration. NTs are known to modulate the development and synaptic efficacy of neurotransmitter systems that are affected by DR. However, it is still unknown whether the actions of NTs in dark-reared animals involve interaction with neurotransmitter systems. We have studied the effects of DR on the expression of key molecules in the glutamatergic and GABAergic systems in control and NT-treated animals. We have found that DR reduced the expression of the NMDA receptor 2A subunit and its associated protein PSD-95 (postsynaptic density-95), of GRIP (AMPA glutamate receptor interacting protein), and of the biosynthetic enzyme GAD (glutamic acid decarboxylase).

Returning dark-reared animals to light for $2 \mathrm{hr}$ restored normal expression of the above-mentioned proteins almost completely. NT treatment specifically counteracts DR effects; NGF acts primarily on the NMDA system, whereas BDNF acts primarily on the GABAergic system. Finally, the action of NT4 seems to involve both excitatory and inhibitory systems. These data demonstrate that different NTs counteract DR effects by modulating the expression of key molecules of the excitatory and inhibitory neurotransmitter systems.

Key words: neurotrophins; GAD; NMDAR; PSD-95; GRIP; dark rearing; visual experience; visual cortex

\section{Introduction}

Total lack of visual experience strongly affects the development of vision; the visual system remains primarily immature even after the end of the critical period (Timney et al., 1978; Fagiolini et al., 1994), as shown by the low visual acuity, large cortical receptivefield size, and poor selectivity for orientation found in the visual cortex of dark-reared (DR) animals. DR also prolongs the critical period of visual cortical plasticity in response to monocular deprivation and delays the developmental decrease of long-term potentiation (LTP) in the visual cortex (Kirkwood et al., 1995).

Recent data demonstrate that total lack of visual experience impairs neurotrophin (NT) signaling (Viegi et al., 2002). NTs are in the position to act as mediators and modulators of electrical activity in regulating visual cortical development. NTs are produced and released in an activity-dependent manner; their expression in the visual cortex depends on visual experience (Castren et al., 1992; Blochl and Thoenen, 1995; Bozzi et al., 1995), and they control the development of the visual system (Berardi et al., 1994; Berardi and Maffei, 1999; McAllister, 1999). In particular, blockade of endogenous NGF impairs development of vi-

Received Sept. 23, 2002; revised Feb. 11, 2003; accepted Feb. 19, 2003.

This work was supported by European Economic Community BioMed Contract BMH4-CT96-1604, Consiglio Nazionale delle Ricerche targeted project Biotechnology SP5, and Ministero dell'Universitá e della Ricerca Scientifica e Tecnologica, Programma di Ricerca di Interesse Nazionale (COFIN 2000/2002). Y.B. was supported by Telethon Fellowship 461/bi. We thank Dr. D. Mercanti for supplying NGF and Regeneron Pharmaceuticals for supplying human recombinant NT4 and BDNF.

Correspondence should be addressed to Dr. Alessandro Viegi, Istituto di Neuroscienze, Consiglio Nazionale delle Ricerche, Moruzzi 1, 56100 Pisa, Italy. E-mail: viegi@in.pi.cnr.it.

Copyright $\odot 2003$ Society for Neuroscience $\quad$ 0270-6474/03/233566-06\$15.00/0 sual acuity and prolongs the critical period (Berardi et al., 1994; Domenici et al., 1994). In contrast, increased availability of NTs counteracts the effects of DR. NT mechanisms of action in visual cortical development, both physiological and in the case of absent visual experience, are still unclear. It has been demonstrated recently that NTs influence synaptic transmission and plasticity (McAllister, 1999; Poo, 2001), and that NT availability regulates the development of neurotransmitter systems in the visual cortex (Huang et al., 1999). It is conceivable to hypothesize that NTs could modulate DR effects by exerting their action on neurotransmitter systems. We therefore explored neurotransmitter systems as possible targets of NT action in dark-reared animals, studying the expression of synaptic molecules in the excitatory and inhibitory circuitry in the visual cortex of DR rats, DR rats briefly exposed to light, and DR rats after exogenous supply of NTs (NGF, BDNF, and NT4). Among these molecules, we studied glutamate receptors, proteins promoting the adequate clustering and targeting of glutamatergic receptors, and the GABA biosynthetic enzyme glutamic acid decarboxylase (GAD). We found that lack of sensory experience reduces the expression of all these synaptic components in the visual cortex, and that each of the exogenously supplied NTs is able to counteract these effects in a specific manner.

\section{Materials and Methods}

Animal treatment. All animal experiments were performed according to the guidelines in ophthalmic and vision research provided by the Association for Research in Vision and Ophthalmology and approved by the Italian Ministry of Public Health. 
Long-Evans rats $(n=41)$ were kept with their mother from birth to postnatal day 30 (P30) in a dark room. Some of the animals were reexposed to light for $2 \mathrm{hr}$ at P30. For NT treatment, osmotic minipumps, connected to a stainless-steel cannula, were implanted at P23 under avertin anesthesia in the visual cortex ( $1 \mathrm{~mm}$ lateral to lambda) of dark-reared rats. The minipumps were filled with NGF $(1 \mu \mathrm{g} / \mu \mathrm{l}$; a kind gift from Dr. D. Mercanti, Consiglio Nazionale delle Ricerche, Rome, Italy), human recombinant BDNF ( $5 \mu \mathrm{g} / \mu \mathrm{l}$; Regeneron Pharmaceuticals, Tarrytown, NY), or NT4 ( $1 \mu \mathrm{g} / \mu \mathrm{l}$; Regeneron Pharmaceuticals). Nonspecific effects of implant were checked with infusion of cytochrome C (CYT, $5 \mu \mathrm{g} / \mu \mathrm{l}$; Sigma, St. Louis, MO). After implant, animals were returned to the dark room until P30. Light-reared (LR) animals were either left untreated $(n=15)$ or infused with NTs or CYT for 1 week (from P23 to P30; $n=$ 12) to evaluate the effect of NTs on the visual cortex of animals reared under normal conditions.

Immunoblotting and densitometry. Proteins were extracted from visual cortices with lysis buffer (1\% Triton X-100, 10\% glycerol, $20 \mathrm{~mm}$ Tris$\mathrm{HCl}, \mathrm{pH}$ 7.5, $150 \mathrm{~mm} \mathrm{NaCl}, 10 \mathrm{~mm}$ EDTA, $0.1 \mathrm{~mm} \mathrm{Na}_{3} \mathrm{VO}_{4}, 1 \mu \mathrm{g} / \mathrm{ml}$ leupeptin, $1 \mu \mathrm{g} / \mathrm{ml}$ aprotinin, and $1 \mathrm{~mm}$ PMSF), and the total concentration of the samples was assessed with a protein assay kit (Bio-Rad, Hercules, CA) using a BSA-based standard curve. Ten micrograms of proteins were electrophoresed with 10\% SDS-PAGE and transferred to nitrocellulose. Blots hybridized with monoclonal antibodies were blocked with 3\% BSA (Sigma) and 0.15\% Tween 20 in TBS for $2 \mathrm{hr}$ and incubated with anti-postsynaptic density (PSD)-95 (Upstate Biotechnology, Lake Placid, NY) and anti-glutamate receptor-interacting protein (GRIP) (Transduction Laboratories, Lexington, KY), diluted at $1 \mu \mathrm{g} / \mathrm{ml}$ in TBS with $2 \%$ BSA and $0.1 \%$ Tween 20 . Blots hybridized with polyclonal antibodies were blocked with $4 \%$ dry milk powder (Bio-Rad) and $0.2 \%$ Tween 20 in TBS for $2 \mathrm{hr}$ and incubated overnight at $4^{\circ} \mathrm{C}$ with anti-NMDA receptor 2A (NR2A) (Upstate Biotechnology) and antiGAD65/67 (Chemicon, Temecula, CA), all diluted at $1 \mu \mathrm{g} / \mathrm{ml}$ in TBS, $2 \%$ milk, and $0.1 \%$ Tween 20 . After washing, blots were incubated for $1 \mathrm{hr}$ at $30^{\circ} \mathrm{C}$ with HRP-conjugated secondary antibody $(0.3 \mu \mathrm{g} / \mathrm{ml}$ goat antimouse for anti PSD-95 and anti-GRIP; $0.3 \mu \mathrm{g} / \mathrm{ml}$ goat anti-rabbit for anti-NR2A and anti-GAD65/67; Bio-Rad), developed by the ECL system (Amersham Biosciences, Little Chalfont, UK), and captured on autoradiographic films.

To account for loading errors, all filters were stripped with Re-Blot (Chemicon), blocked with $4 \%$ dry milk powder and $0.2 \%$ Tween 20 in TBS for $2 \mathrm{hr}$, and reprobed overnight at $4^{\circ} \mathrm{C}$ with anti-glucose-6phosphate dehydrogenase (G-6-PDH; diluted $1 \mu \mathrm{g} / \mathrm{ml}$ in TBS, $2 \%$ milk, and $0.1 \%$ Tween 20; Sigma). The following day, blots were incubated with HRP-conjugated secondary antibody (goat anti-rabbit) as described for other antibodies.

Films were digitized with a camera and band optical densities (ODs) relative to the proteins of interest, and corresponding G-6-PDH internal standards were measured with Microcomputer Imaging Device software (Imaging Research, St. Catharines, Ontario, Canada). For each sample (corresponding to the visual cortex of a single animal), we calculated the ratio of protein to G-6-PDH OD values, and these numbers were used to calculate the mean \pm SEM. Finally, these values were normalized with respect to a LR control run on the same gel, and results were presented as a percentage of LR values.

\section{Results}

We investigated whether total lack of visual experience affects the expression of specific synaptic proteins in the inhibitory and excitatory systems and whether NTs counteract DR effects. We used Western blot analysis to determine changes in the level of expression of NR2A subunit and PSD-95 in the NMDA system, of GRIP in the AMPA system, and of GAD for the inhibitory system. By means of densitometric analysis of immunoblot films, we quantitatively evaluated DR effects on these molecules in protein extracts prepared from the visual cortex of P30 LR and DR animals, P30 DR animals briefly re-exposed to light, and P30 DR animals after exogenous supply of NTs.

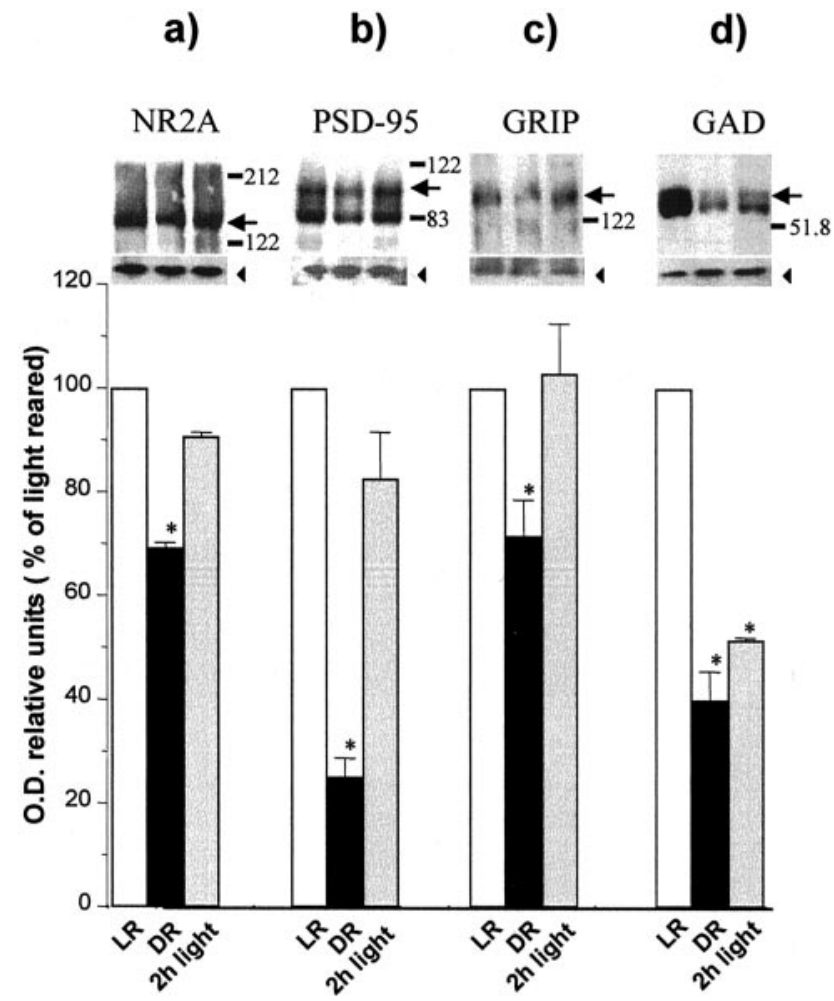

Figure 1. DR reduces the expression of synaptic proteins in the glutamatergic and GABAergic system, and visual experience rapidly restores the glutamatergic system. $a-d$, Quantitative immunoblotting was performed on protein extracts prepared from visual cortices of $L R$ rats $(n=12)$, DR rats $(n=11)$, or DR rats exposed to light for $2 \mathrm{hr}(n=3)$ for NR2A (a), PSD-95 (b), $\operatorname{GRIP}(c)$, and GAD ( $d$ ) (asterisks indicate significant difference vs $L R ; t$ test; $p<0.05$ ). Data are presented as percentage of $O D$ values measured in $L R$ rats (mean $\pm S E M$ ). Arrows indicate bands relative to the proteins of interest; arrowheads indicate G-6-PDH.

\section{Dark rearing reduces the expression of molecules in the} excitatory and inhibitory system

\section{NR2A}

For the NMDA receptor system, we analyzed the NR2A subunit, known to be affected by sensory experience (Quinlan et al., 1999; Philpot et al., 2001). In accordance with Quinlan et al. (1999), we found that NR2A levels were significantly decreased in DR compared with normal animals by $\sim 30 \%$ (Fig. $1 a$ ) (mean normalized OD for LR animals, 100, $n=12$; for DR animals, $69.3 \pm 0.94, n=$ $11 ; t$ test; $p<0.05)$.

\section{PSD-95}

PSD-95 is a protein associated with the NMDA receptor complex, proposed to be important in coupling NMDA receptors to pathways that control synaptic plasticity (Migaud et al., 1998). We looked at the experience-dependent expression of PSD-95 and found that DR influenced its levels even more than those of the NR2A subunit (Fig. 1b) (mean normalized OD for LR animals, $100, n=12$; for DR animals, $25.14 \pm 3.6, n=11$; $t$ test; $p<0.05$ ).

\section{GRIP}

AMPA receptors have been unquestionably implicated as important players in synaptic plasticity (Carroll et al., 2001). We found that lack of visual experience causes a decline in GRIP expression to $72 \%$ of the LR value (Fig. 1c) (mean normalized OD for LR animals, $100, n=8$; for DR animals, $71.6 \pm 6.9, n=5$; $t$ test; $p<0.05$ ). 


\section{GAD65/67}

The maturation of the inhibitory system is critical for the development of visual cortical properties and plasticity (Hensch et al., 1998; Fagiolini and Hensch, 2000) and is crucial for critical period closure (Huang et al., 1999). To investigate the effects of DR on the GABAergic system, we assessed the expression of the GABAsynthesizing enzyme GAD. We found that DR reduces GAD expression by $\sim 60 \%$ (Fig. 1d) (mean normalized OD for LR animals, $100, n=12$; for DR animals, $40 \pm$ 5.4, $n=11$; $t$ test; $p<0.05)$.

Visual experience rapidly restores the expression of synaptic proteins in the excitatory system but not the expression of GAD65/67

A brief re-exposure to light is sufficient to rescue visual properties in DR animals (Buisseret et al., 1978; Cynader, 1983) and re-establish normal levels of expression of NR2A subunit in the visual cortex (Quinlan et al., 1999; Philpot et al., 2001). To evaluate whether sensory experience might rapidly restore the effects of DR on the expression levels of all synaptic proteins examined, DR animals were exposed to light for $2 \mathrm{hr}$. This brief exposure was sufficient to completely rescue the deficit observed for NR2A (Fig. 1a) (mean normalized OD for DR plus $2 \mathrm{hr}$ light, $90.7 \pm$ 0.75, $n=3$; LR, 100, $n=12$ ), PSD-95 (Fig. 1b) (mean normalized OD for DR plus 2 hr light, $82.6 \pm 8.9, n=3$; LR, 100, $n=$ 12), GRIP (Fig. 1c) (mean normalized OD for DR plus $2 \mathrm{hr}$ light, $102.9 \pm 9.6, n=2$; LR, 100, $n=8$ ). GAD65/67 protein levels were not substantially affected by this brief visual experience (Fig. 1d) (mean normalized OD for DR plus $2 \mathrm{hr}$ light, $51.5 \pm 0.5, n=3$; LR, 100, $n=12$; $p<0.05)$.

\section{NGF, BDNF, and NT4 differentially rescue dark rearing} effects on the glutamatergic and GABAergic system

We know that NTs counteract the effects of DR (Fagiolini et al., 1997; Pizzorusso et al., 2000). Given the known influence of NTs on synaptic transmission (Poo, 2001), we investigated whether NTs might distinctively rescue the expression of the synaptic proteins of the excitatory and the inhibitory system analyzed so far. We administered NTs in the visual cortex of DR rats by means of osmotic minipumps from P23 to P30 and then assessed the expression of NR2A, PSD-95, GRIP, and GAD65/67 as described previously.

As shown in Figure $2 a$, NGF seems to be able to counteract the DR effect on NR2A subunit expression; NR2A levels in NGFtreated DR animals are not significantly different from those found in LR ones (mean normalized OD for DR plus NGF, $91.1 \pm 2.1, n=9 ; \mathrm{LR}, 100, n=12)$. This effect is not caused by the procedure of minipump implant, because it is absent in CYTtreated rats (mean normalized OD for DR plus CYT, 68.2 \pm 2.1 , $n=4)$. BDNF and NT4 exogenous supply does not restore NR2A expression to normal levels (mean normalized OD for DR plus BDNF, $65.3 \pm 1.55, n=6$; DR plus NT4, $52.6 \pm 12.6, n=3$ ).
NGF is also able to attenuate the effect produced by DR on PSD-95 expression (Fig. 2b) (mean normalized OD for DR plus NGF, 50.2 $\pm 2.6, n=9$; LR, 100, $n=12$ ). BDNF does not show any effect on PSD-95 (Fig. $2 b$ ) (mean normalized OD for DR plus BDNF, $24.6 \pm 6.8, n=6$ ), whereas NT4 has effects comparable with those of NGF (Fig. 2b) (mean normalized OD for DR plus NT4, $63.8 \pm 4.8, n=6)$. Also, in this case, CYT treatment is completely ineffective (OD for DR plus CYT, $26 \pm 5.5, n=4$ ).

All NTs appear to exert a role in rescuing the DR-induced decrease in GRIP expression. In particular, NGF and BDNF show a strong effect. When they are exogenously supplied, GRIP expression reaches higher levels than those present in age-matched LR controls (Fig. 2c) (mean normalized OD for DR plus NGF, 182.4 $\pm 19.5, n=3$; OD for DR plus BDNF, $217.47 \pm 4.8, n=3$; OD for LR, 100, $n=8$ ). GRIP levels go back to their physiological values by infusing NT4 (Fig. 2c) (mean normalized OD for DR plus NT4, $97.2 \pm 14, n=3$ ). No effect was observed in CYTtreated animals (OD for DR plus CYT, 74.6 \pm 9.7, $n=4$ ).

We found that BDNF reverses the effects of DR on GAD65/67 levels, bringing them back to LR values (Fig. $2 d$ ) (mean normalized OD for DR plus BDNF, 86.1 $\pm 6.2, n=6$; OD for LR, 100, $n=12)$. Also, NT4 shares tropomyosin-related kinase B (TrkB) receptors with BDNF and shows a similar rescuing effect (Fig. 2d) (mean normalized OD for DR plus NT4, $116.3 \pm 4.1, n=3$ ). Conversely, NGF does not at all influence GAD65/67 expression (Fig. 2d) (mean normalized OD for DR plus NGF, 41.5 $\pm 2.9, n=$ 
Table 1. Effects of NTs on different synaptic proteins in the visual cortex of DR rats

\begin{tabular}{llll}
\hline & NGF & BDNF & NT4 \\
\hline NR2A & + & - & - \\
PSD-95 & + & - & + \\
GRIP & ++ & ++ & + \\
GAD & - & + & + \\
\hline
\end{tabular}

9). Again, CYT treatment is ineffective (OD for DR plus CYT, $48.3 \pm 9.5, n=4)$.

The specific effects of NGF, BDNF, and NT4 on the expression of the synaptic molecules examined in DR rats are summarized in Table 1.

\section{NT infusion does not influence the expression of NR2A,} GRIP, PSD-95, and GAD65/67 in the visual cortex of LR rats To investigate whether NT administration per se in LR animals could modulate the expression of the proteins analyzed, we infused NGF, BDNF, NT4, and CYT (as a control) in LR rats $(n=$ 3 animals per treatment group). We found that expression of NR2A, PSD-95, GRIP, and GAD did not show any statistically significant difference with respect to the controls. Mean \pm SEM OD values (normalized to CYT-treated cortices, 100\%) in LR animals treated with NGF, BDNF, or NT4 were, respectively: NR2A, $103.5 \pm 13.8,97.9 \pm 10.5,107.5 \pm 26.1$; PSD-95, $95.1 \pm$ $11.9,96.1 \pm 10.5,91.7 \pm 2.7$; GRIP, $102.9 \pm 23.7,92.4 \pm 4.2$, $93.9 \pm 9.1 ; \mathrm{GAD} 65 / 67,92.7 \pm 7.4,99.9 \pm 10.7,100.6 \pm 8.4$.

\section{Discussion}

\section{Dark rearing reduces the expression of synaptic proteins in} the glutamatergic and GABAergic systems

Rearing animals in complete darkness from birth provides a useful way to study how sensory experience exerts an influence on the physiological, anatomical, and molecular properties of the visual system. In this experimental paradigm, we observed a decreased expression of all synaptic molecules examined. The decrease could be a general consequence of the total absence of sensory input caused by the delayed maturation of visual cortex induced by DR. Alternatively, it might represent the cause of at least some of the effects observed in DR rats. Although our results cannot discriminate between these two possibilities, they point out that the expression of different sets of molecules is regulated by visual experience.

It has been proposed that sensory deprivation acts on the experience-dependent rearrangements of synaptic connections required to form precise sensory maps (Lendvai et al., 2000). Under this light, the study of molecular changes occurring at synaptic sites could provide an explanation for the modulation of synaptic strength that is crucial for synaptic plasticity.

\section{Visual experience rapidly restores the expression of synaptic proteins in the excitatory system but not the expression of GAD65/67}

A brief visual experience after DR is able to restore the expression of the examined molecules in the glutamatergic system at values comparable with those found in the visual cortex of age-matched LR animals. This has been observed previously by Quinlan et al. (1999) for NMDA NR2A subunit, but it occurs also for PSD-95 and GRIP.

Interestingly, the same brief light exposure does not restore GAD65/67 expression in DR rats. A possible explanation is that because the inhibitory system maturation is slower than that of the excitatory system, the GABAergic system of DR rats could be not ready to promptly respond to sensory experience. Indeed, Morales et al. (2002) showed that the reduction of IPSC magnitude observed in DR rats was reversed after a time as long as $2 \mathrm{~d}$ of exposure to light. These data suggest that the experiencedependent rescue of GABAergic function after DR is a slow process, which is likely to require the resynthesis of key molecules of GABA-mediated neurotransmission (such as receptors and biosynthetic enzymes). However, it could also be possible that the postsynaptic machinery (involving NR2A, PSD-95, and GRIP) responds faster than the presynaptic element to those changes that are associated with a brief alteration of sensory experience.

Dark rearing influences the expression of synaptic proteins in the glutamatergic and GABAergic systems by a specific neurotrophin-dependent mechanism

The results reported demonstrate that visual experience regulates the expression levels of the glutamatergic and GABAergic molecules examined. We tested the involvement of NTs in controlling these events. We found that NT infusion in DR animals affects in a specific manner both the glutamatergic and the GABAergic synaptic proteins. In contrast, administration of NTs in the visual cortex of LR animals did not alter the expression of NR2A, PSD95, GRIP, and GAD65/67. This suggests that the increase in NR2A, PSD-95, GRIP, and GAD65/67 expression obtained with NT supply to DR animals is attributable to compensation of a deficit in endogenous NT function induced by DR, rather than to a general ability of NTs to upregulate the expression of these molecules. Indeed, it is known that DR strongly downregulates signal transduction pathways of NTs (Viegi et al., 2002). Thus, exogenous administration of NTs in DR animals would contribute to restore normal levels of NT-mediated signaling. In animals reared under conditions of normal visual experience, NT function is normal, and visual cortical neurons might not be prone to increase the expression of specific molecules of neurotransmitter systems in response to increased NT levels. Indeed, data from the literature indicate that the effects of the exogenous supply of NT are primarily directed on neuropeptide expression, and few examples of effects on synaptic receptors are reported. BDNF and NT4 upregulate neuropeptide expression in the dopaminergic system (Arenas et al., 1996), whereas in the visual cortex, BDNF and NT4 upregulate NPY expression (Croll et al., 1994; Nawa et al., 1994; Lodovichi et al., 2000). BDNF also modulates AMPA but not NMDA receptors in neocortical neurons (Narisawa-Saito et al., 1999).

NGF

NGF seems to be the elective trophic factor for the NMDA system. In fact, it is able to recover the effect produced by DR on NR2A and PSD-95 expression. We could suppose that the effect of NGF on NR2A expression in DR rats might affect LTP in the visual cortex. In the rat visual cortex, LTP is maximally expressed after eye opening and progressively downregulated thereafter to disappear after P30, in perfect timing with the closure of the critical period. Pesavento et al. (2000) showed that LTP expression is blocked by local application of exogenous NGF at an early stage of postnatal development. Interestingly, there exists a connection between the developmentally regulated LTP and PSD-95. Migaud et al. (1998) found that in hippocampal slices from PSD-95 mutant mice, some components of the signaling complex that elicit LTP and trigger long-term depression are lost. The decreased PSD-95 and NR2A expression is probably the reason why DR animals show a retarded loss of LTP during development. NGF also exerts a strong action on the key molecule GRIP, 
which is involved in AMPA recycling. This result should not be surprising in light of the "silent synapse" hypothesis. One of the mechanisms proposed to explain the silent synapse hypothesis for the expression of synaptic potentiation is based on continuous recycling of AMPA receptors in and out from the synaptic membrane (Liao et al., 1995). According to this hypothesis, NMDAs and AMPAs should communicate with each other to decide the right time for the switch from silent to functional synapses. In fact, changes in NMDA receptor properties may have a large impact on experience-dependent regulation of AMPA receptormediated responses (Hayashi et al., 2000).

\section{$B D N F$}

BDNF exogenous administration does not have any effect on the expression of proteins belonging to the NMDA receptor complex (NR2A and PSD-95). BDNF, as well as NGF, produces an overshoot of GRIP expression. Because NMDA and AMPA systems are functionally (Hayashi et al., 2000) and structurally (Tu et al., 1999) correlated, we can suppose that NGF exerts its role on GRIP by acting on the NMDA system, whereas BDNF acts more directly on the AMPA system without interfering in NMDA receptor complex expression.

The role of BDNF on the GABAergic system is already well recognized. BDNF acts on GABAergic differentiation (Marty et al., 1996). BDNF has been shown to promote GABA release from cortical synaptosomes (Sala et al., 1998) and in transgenic mice overexpressing BDNF; there is an accelerated rate of maturation of the GABAergic system, which is accompanied by a premature end of the critical period (Huang et al., 1999). Our demonstration that exogenous administration of BDNF in the visual cortex of DR rats during the critical period can restore normal levels of GAD65/67 supports the notion that BDNF is crucially involved in the regulation of the GABAergic system.

In addition, GRIP1 was surprisingly found in GABAergic synapses, indicating that it might regulate both excitatory and inhibitory synaptic function (Dong et al., 1997). These evidences support the hypothesis that the effects of BDNF on the modulation of GRIP expression pass through the AMPA and/or the GABAergic system. In this respect, it would be of extreme interest to investigate whether BDNF-mediated upregulation of GRIP is restricted only to GABA-positive neurons in the visual cortex of DR rats.

The BDNF-dependent upregulation of both GRIP and GAD observed in the visual cortex of DR rats suggests that BDNF could act in a dual way to modulate cortical inhibition, by regulating the expression of key molecules of both the AMPA and GABA system expressed by GABA neurons. Indeed, recent work showed that BDNF is involved in synapse scaling, a homeostatic process of synaptic plasticity originally described in cultured cortical networks (Turrigiano et al., 1998) and shown recently to be present also in the developing visual cortex in vivo (Desai et al., 2002). In particular, it has been proposed that in cultured cortical networks, synaptic scaling occurs through the activity-dependent release of BDNF from pyramidal cells, which results in GABA neuron activation to maintain the balance between cortical excitation and inhibition (Rutherford et al., 1998).

\section{NT4}

Our results demonstrate that NT4 regulates the expression of key molecules of both the excitatory and inhibitory system. NT4 exogenous administration counteracts DR effects on PSD-95 but not on NR2A in the NMDA system. Surprisingly, even if an NT4dependent increase in PSD-95 expression does not reach the LR control values, its effect is larger than that produced by NGF.
NT4, like BDNF, also counteracts DR-induced downregulation of GRIP and GAD. This effect could be caused by signaling pathways downstream to the TrkB receptor, shared by NT4 and BDNF. We speculate that both endogenous TrkB ligands could act on the same neurotransmitter systems (i.e., AMPA and GABA) to modulate plastic modification of visual cortical circuitry during the critical period. Indeed, TrkB signaling is clearly involved in ocular dominance column formation (Cabelli et al., $1995,1997)$ and is necessary for the expression of LTP in the visual cortex during postnatal development (Sermasi et al., 2000).

\section{References}

Arenas E, Akerud P, Wong V, Boylan C, Persson H, Lindsay RM, Altar CA (1996) Effects of BDNF and NT-4/5 on striatonigral neuropeptides or nigral GABA neurons in vivo. Eur J Neurosci 8:1707-1717.

Berardi N, Maffei L (1999) From visual experience to visual function: roles of neurotrophins. J Neurobiol 41:119-126.

Berardi N, Cellerino A, Domenici L, Fagiolini M, Pizzorusso T, Cattaneo A, Maffei L (1994) Monoclonal antibodies to nerve growth factor affect the postnatal development of the visual system. Proc Natl Acad Sci USA 91:684-688.

Blochl A, Thoenen H (1995) Characterization of nerve growth factor (NGF) release from hippocampal neurons: evidence for a constitutive and an unconventional sodium-dependent regulated pathway. Eur J Neurosci 7:1220-1228.

Bozzi Y, Pizzorusso T, Cremisi F, Rossi FM, Barsacchi G, Maffei L (1995) Monocular deprivation decreases the expression of messenger RNA for brain-derived neurotrophic factor in the rat visual cortex. Neuroscience 69:1133-1144.

Buisseret P, Gary-Bobo E, Imbert M (1978) Ocular motility and recovery of orientational properties of visual cortical neurones in dark-reared kittens. Nature 272:816-817.

Cabelli RJ, Hohn A, Shatz CJ (1995) Inhibition of ocular dominance column formation by infusion of NT-4/5 or BDNF. Science 267:1662-1666.

Cabelli RJ, Shelton DL, Segal RA, Shatz CJ (1997) Blockade of endogenous ligands of trkB inhibits formation of ocular dominance columns. Neuron 19:63-76.

Carroll RC, Beattie EC, von Zastrow M, Malenka RC (2001) Role of AMPA receptor endocytosis in synaptic plasticity. Nat Rev Neurosci 2:315-324.

Castren E, Zafra F, Thoenen H, Lindholm D (1992) Light regulates expression of brain-derived neurotrophic factor mRNA in rat visual cortex. Proc Natl Acad Sci USA 89:9444-9448.

Croll SD, Wiegand SJ, Anderson KD, Lindsay RM, Nawa H (1994) Regulation of neuropeptides in adult rat forebrain by the neurotrophins BDNF and NGF. Eur J Neurosci 6:1343-1353.

Cynader M (1983) Prolonged sensitivity to monocular deprivation in darkreared cats: effects of age and visual exposure. Brain Res 284:155-164.

Desai NS, Cudmore RH, Nelson SB, Turrigiano GG (2002) Critical periods for experience-dependent synaptic scaling in visual cortex. Nat Neurosci 5:783-789.

Domenici L, Cellerino A, Berardi N, Cattaneo A, Maffei L (1994) Antibodies to nerve growth factor (NGF) prolong the sensitive period for monocular deprivation in the rat. NeuroReport 5:2041-2044.

Dong H, O’Brien RJ, Fung ET, Lanahan AA, Worley PF, Huganir RL (1997) GRIP: a synaptic PDZ domain-containing protein that interacts with AMPA receptors. Nature 386:279-284.

Fagiolini M, Hensch TK (2000) Inhibitory threshold for critical-period activation in primary visual cortex. Nature 404:183-186.

Fagiolini M, Pizzorusso T, Berardi N, Domenici L, Maffei L (1994) Functional postnatal development of the rat primary visual cortex and the role of visual experience: dark rearing and monocular deprivation. Vision Res 34:709-720.

Fagiolini M, Pizzorusso T, Porciatti V, Cenni M, Maffei L (1997) Transplant of Schwann cells allows normal development of the visual cortex of darkreared rats. Eur J Neurosci 9:102-112.

Hayashi Y, Shi SH, Esteban JA, Piccini A, Poncer JC, Malinow R (2000) Driving AMPA receptors into synapses by LTP and CaMKII: requirement for GluR1 and PDZ domain interaction. Science 287:2262-2267.

Hensch TK, Fagiolini M, Mataga N, Stryker MP, Baekkeskov S, Kash SF (1998) Local GABA circuit control of experience-dependent plasticity in developing visual cortex. Science 282:1504-1508. 
Huang ZJ, Kirkwood A, Pizzorusso T, Porciatti V, Morales B, Bear MF, Maffei L, Tonegawa S (1999) BDNF regulates the maturation of inhibition and the critical period of plasticity in mouse visual cortex. Cell 98:739-755.

Kirkwood A, Lee HK, Bear MF (1995) Co-regulation of long-term potentiation and experience-dependent synaptic plasticity in visual cortex by age and experience. Nature 375:328-331.

Lendvai B, Stern EA, Chen B, Svoboda K (2000) Experience-dependent plasticity of dendritic spines in the developing rat barrel cortex in vivo. Nature 404:876-881.

Liao D, Hessler NA, Malinow R (1995) Activation of postsynaptically silent synapses during pairing-induced LTP in CA1 region of hippocampal slice. Nature 375:400-404.

Lodovichi C, Berardi N, Pizzorusso T, Maffei L (2000) Effects of neurotrophins on cortical plasticity: same or different? J Neurosci 20:2155-2165.

Marty S, Berninger B, Carroll P, Thoenen H (1996) GABAergic stimulation regulates the phenotype of hippocampal interneurons through the regulation of brain-derived neurotrophic factor. Neuron 16:565-570.

McAllister AK (1999) Subplate neurons: a missing link among neurotrophins, activity, and ocular dominance plasticity? Proc Natl Acad Sci USA 96:13600-13602.

Migaud M, Charlesworth P, Dempster M, Webster LC, Watabe AM, Makhinson M, He Y, Ramsay MF, Morris RG, Morrison JH, O’Dell TJ, Grant SG (1998) Enhanced long-term potentiation and impaired learning in mice with mutant postsynaptic density-95 protein. Nature 396:433-439.

Morales B, Choi SY, Kirkwood A (2002) Dark rearing alters the development of the GABAergic transmission in visual cortex. J Neurosci 22:8084-8090.

Narisawa-Saito M, Carnahan J, Araki K, Yamaguchi T, Nawa H (1999) Brain-derived neurotrophic factor regulates the expression of AMPA receptor proteins in neocortical neurons. Neuroscience 88:1009-1014.

Nawa H, Pelleymounter MA, Carnahan J (1994) Intraventricular administration of BDNF increases neuropeptide expression in newborn rat brain. J Neurosci 14:3751-3765.

Pesavento E, Margotti E, Righi M, Cattaneo A, Domenici L (2000) Blocking the NGF-TrkA interaction rescues the developmental loss of LTP in the rat visual cortex: role of the cholinergic system. Neuron 25:165-175.

Philpot BD, Sekhar AK, Shouval HZ, Bear MF (2001) Visual experience and deprivation bidirectionally modify the composition and function of NMDA receptors in visual cortex. Neuron 29:157-169.

Pizzorusso T, Fagiolini M, Gianfranceschi L, Porciatti V, Maffei L (2000) Role of neurotrophins in the development and plasticity of the visual system: experiments on dark rearing. Int J Psychophysiol 35:189-196.

Poo MM (2001) Neurotrophins as synaptic modulators. Nat Rev Neurosci 2:24-32.

Quinlan EM, Philpot BD, Huganir RL, Bear MF (1999) Rapid, experiencedependent expression of synaptic NMDA receptors in visual cortex in vivo. Nat Neurosci 2:352-357.

Rutherford LC, Nelson SB, Turrigiano GG (1998) BDNF has opposite effects on the quantal amplitude of pyramidal neuron and interneuron excitatory synapses. Neuron 21:521-530.

Sala R, Viegi A, Rossi FM, Pizzorusso T, Bonanno G, Raiteri M, Maffei L (1998) Nerve growth factor and brain-derived neurotrophic factor increase neurotransmitter release in the rat visual cortex. Eur J Neurosci 10:2185-2191.

Sermasi E, Margotti E, Cattaneo A, Domenici L (2000) Trk B signalling controls LTP but not LTD expression in the developing rat visual cortex. Eur J Neurosci 12:1411-1419.

Timney B, Mitchell DE, Giffin F (1978) The development of vision in cats after extended periods of dark-rearing. Exp Brain Res 31:547-560.

Tu JC, Xiao B, Naisbitt S, Yuan JP, Petralia RS, Brakeman P, Doan A, Aakalu VK, Lanahan AA, Sheng M, Worley PF (1999) Coupling of mGluR/ Homer and PSD-95 complexes by the Shank family of postsynaptic density proteins. Neuron 23:583-592.

Turrigiano GG, Leslie KR, Desai NS, Rutherford LC, Nelson SB (1998) Activity-dependent scaling of quantal amplitude in neocortical neurons. Nature 391:892-896.

Viegi A, Cotrufo T, Berardi N, Mascia L, Maffei L (2002) Effects of dark rearing on phosphorylation of neurotrophin Trk receptors. Eur J Neurosci 16:1925-1930. 